Débridement akuter und chronischer Wunden

\title{
Monofilament-Kompresse beseitigt Biofilm
}

— Die Sanierung des Wundbettes durch das Entfernen nekrotischer und fibrinöser Beläge ist eine Grundvoraussetzung für eine erfolgreiche Wundheilung. Besonders chronische Wunden, die oft bakterielle Biofilme aufweisen, sind eine Herausforderung. Mit Hilfe einer Monofilament-Kompresse kann die bakterielle Keimbelastung effektiv reduziert werden, wie Prof. Gregory Schultz, Gainesville/USA kürzlich in London berichtete.

Eine kontrollierte Entzündung, wie sie als Phase der natürlichen Wundheilung auftritt, begünstigt die Heilung, da die Immunzellen planktonische Bakterien abtöten und über eine gesteigerte Proteasenproduktion denaturierte Komponenten der Extrazellulärmatrix abbauen, erinnerte Schultz. Gerät die Entzündung jedoch außer Kontrolle und wird chronisch, dann wirke sich die verstärkte Aktivität der Proteasen negativ aus, und sie zerstörten die für den Heilungsverlauf relevanten Proteine. Gegen bakterielle Biofilme seien die Entzündungszellen machtlos. „Biofilme treten wahrscheinlich in $90 \%$ der chronischen Wunden auf und beeinträchtigen die Wundheilung", so Schultz. Die Entfernung des nekrotischen Gewebes, des Biofilms und die Reduzierung der Keimbelastung seien daher entscheidende Komponenten der Wundbettvorbereitung.

Die Monofilamentfaser-Kompresse Debrisoft ${ }^{\circledR}$ bestehe aus Tausenden synthetischer Fasern mit konisch zulaufenden Enden und eigne sich besonders gut zum Débridement, da sie effektiv nekrotisches Gewebe entferne sowie die bakterielle Keimbelastung minimiere und dabei wenig Schmerzen verursache. Schultz präsentierte zudem eigene Untersuchungen, in denen das Débridement mit der Kompresse die bakterielle Keimbelastung eines reifen Pseudomonas aeruginosa-Biofilms auf Schweinehaut-Explantaten um $99 \%$ minderte. Das National Institute for Health and Care Excellance vergab 2014 eine positive Bewertung für den Einsatz der Débridement-Kompresse, da sie sich im Vergleich zu anderen Verfahren (Hydrogel, Mull, Larven) als kosteneffektiv erwiesen habe und weniger Pflegekontakte erfordere, berichtete Andrew Kingsley, Devon/UK.

Symposium „Debridement: Whose problem is it? Solutions for patients, purchasers and providers"; London, 14. Mai 2015 im Rahmen der EWMA 2015 Conference (Veranstalter: Lohmann \& Rauscher)

\section{Belastung im Pflegealltag Wenn es riecht}

— Riecht ein Mensch unangenehm, ist das störend für sein Umfeld, aber auch für den Betroffenen selbst. Jedes Gespräch wird zur Mutprobe und oft mündet die Angst, anderen zu nahe zu kommen, in Einsamkeit. Doch: Den meisten kann geholfen werden - durch die Behandlung der Ursachen und mit dem natürlichen Wirkstoff Chlorophyll (Stozzon ${ }^{\circledast}$ Chlorophyll-Dragees), der Gerüche von innen heraus beseitigt.

Unangenehme Gerüche entstehen unter anderem durch Stoffwechselvorgänge, Krankheiten, Alterungsprozesse oder Fäulnisbakterien in der Mundhöhle. Unbehaglich wird es vor allem in Situationen, in denen man sich besonders nah kommt und nur schwer entziehen kann, zum Beispiel im Pflegealltag oder in den intimen Momenten des Lebens. Dabei macht dem Betroffenen nicht nur der Geruch selbst zu schaffen auch der psychologische Faktor spielt eine Rolle: Die Angst vor Ablehnung ist tief in uns verwurzelt.

Pflegekräfte, die bei Patienten Mund- oder Körpergeruch bemerken, sollten das Thema behutsam ansprechen. Taktgefühl ist also das $\mathrm{A}$ und $\mathrm{O}$, denn niemand möchte vor Dritten bloßgestellt werden:

_ Überlegen Sie, wie man SIE auf Mundoder Körpergeruch ansprechen dürfte, ohne Sie zu kränken.

Neues Design

\section{Ampulle bietet mehr Sicherheit}

_ Fresenius Kabi Deutschland bietet seine bewährten Injektionslösungen ab sofort in einer neuen Ampulle mit optimiertem Design an. Patienten und medizinisches Personal profitieren doppelt: Einerseits vermindert sich zusätzlich ein potentielles mikrobielles Kontaminationsrisiko, andererseits wird die nadelfreie Handhabung vereinfacht. Besonders am neuen Design ist - beispielsweise bei Ampuwa ${ }^{\circledast}$ (Wasser für Injektionszwecke) -, dass die Ampullen im Cluster ausschließlich am Körper und nicht zusätzlich am Ampullenkopf miteinander verbunden sind. Beim Abtrennen aus dem Cluster kann die Ampulle deshalb nicht versehentlich geöffnet werden. Zusätzlich sorgt ein ergonomisch geformter Griff am Abbrechteil des Ampullenkopfes für eine geringere Kontaminationsgefahr beim Abdrehen des Verschlusses. Die geöffnete Fresenius Kabi Ampulle kann auf eine Spritze mit LuerLock-Anschluss aufgeschraubt oder auf eine Spritze mit Luer-Anschluss gesteckt werden. Das Aufziehen erfolgt somit ohne Kanüle und reduziert das Risiko von Nadelstichverletzungen.

www.fresenius-kabi.de
- Suchen Sie das Gespräch unter vier Augen, wenn Sie freundlich fragen, ob Sie etwas Privates ansprechen dürfen. Damit zeigen Sie Respekt und geben dem Patienten die Chance, zuzustimmen oder abzulehnen.

- Verhalten Sie sich als "Anwalt des anderen" und betonen Sie, dass man bei sich selbst Mund- und Körpergeruch nur schwer erkennen und somit jeder betroffen sein kann. Diese Haltung ist wichtig, damit der positive Kontakt bis zum Gesprächsende erhalten bleibt. Ein Hinweis mit Fingerspitzengefühl wird in der Regel von allen Betroffenen leicht verlegen, aber dankbar angenommen.

www.stozzon.de 